Article

\title{
Polyphenols Modulate Alzheimer's Amyloid Beta Aggregation in a Structure-Dependent Manner
}

\author{
Huong T. T. Phan ${ }^{1}$, Kaouthar Samarat ${ }^{1}$, Yuzuru Takamura ${ }^{1}$, Auriane F. Azo-Oussou ${ }^{2}$, \\ Yasutaka Nakazono ${ }^{2}$ and Mun'delanji C. Vestergaard ${ }^{2, *}$ \\ 1 Japan Advanced Institute of Science and Technology, 1-1 Asahidai, Nomi City, Ishikawa 923-1292, Japan; \\ huongptt@hnue.edu.vn (H.T.T.P.); k.samarat@gmail.com (K.S.); takamura@jaist.ac.jp (Y.T.) \\ 2 Department of Food Science and Biotechnology, Kagoshima University, 1-21-24 Korimoto, \\ Kagoshima 890-8580, Japan; oauriane1@gmail.com (A.F.A.-O.); yasutaka33ym@gmail.com (Y.N.) \\ * Correspondence: munde@agri.kagoshima-u.ac.jp; Tel.: +81-99-285-3520
}

Received: 27 February 2019; Accepted: 26 March 2019; Published: 31 March 2019

check for updates

\begin{abstract}
Some polyphenols, which are common natural compounds in fruits, vegetables, seeds, and oils, have been considered as potent inhibitors of amyloid beta $(A \beta)$ aggregation, one critical pathogenic event in Alzheimer's disease (AD). However, the mechanisms by which polyphenols affect aggregation are not fully understood. In this study, we aimed to investigate the effect of two classes of polyphenols (flavonoids and stilbenes) on the self-assembly of $A \beta \_42$, in particular, how this relates to structure. We found that the flavonoids gallocatechin gallate (GCG) and theaflavin (TF) could completely inhibit $A \beta$ aggregation, while two stilbenes, resveratrol and its glucoside derivative piceid, could also suppress $A \beta$ aggregation, but to a much lesser extent. Intriguingly, resveratrol accelerated the formation of $A \beta$ fibrils before its decreasing effect on fibrillation was detected. Atomic force microscopy (AFM) images showed a huge mass of long and thin A $\beta$ fibrils formed in the presence of resveratrol. Although the morphology was the same in the presence of piceid, the fibrils were sparse in the presence of picead. In the presence of flavonoids, A $\beta$ morphology was unchanged from prior to incubation $(0 \mathrm{~h})$, in agreement with amyloid beta kinetics analysis using thioflavin-T fluorescence assay. The electrochemical data showed a higher ability of GCG and TF to interact with $A \beta$ than resveratrol and piceid, which could be attributed to the presence of more aromatic rings and hydroxyl groups. In addition, the two flavonoids exhibited a similar propensity for $A \beta$ aggregation, despite having some differences in their structure. However, in the case of stilbenes, the addition of a glucoside at C-7 slightly decreased anti-A $\beta$ aggregation property compared to resveratrol. These findings contribute to a better understanding of the essential structural features of polyphenols required for inhibiting $A \beta$ aggregation, and the possible mechanisms for modulating aggregation.
\end{abstract}

Keywords: polyphenols; flavonoids; trans-stilbenes; bioactivity; amyloid beta aggregation; modulation

\section{Introduction}

Polyphenols are naturally occurring secondary metabolites that are found in high quantities in fruits, vegetables, seeds, oils, and other foods [1]. They play an essential role in protecting plants from ultraviolet light and against aggression by pathogens or predators, contribute to pigmentation, and facilitate growth and reproduction [2]. More than 8000 natural polyphenols have been identified. Based on the nature of their carbon skeletons, they are grouped into six main categories: flavonoids, phenolic acids and derivatives, stilbenes, curcuminoids, lignans, and tannins [3]. All polyphenols feature more than one phenolic ring with hydroxyl groups in ortho or para positions, which are necessary for redox reactions [4]. These natural compounds exhibit a strong antioxidant power 
due to their properties to scavenge free radicals generated by reactive oxygen species [5] and to chelate highly redox-active metal ions like iron [6,7], thus conferring a protective effect against oxidative damage [8]. Their dietary intake is significantly higher compared to other dietary antioxidants including Vitamins C and E and carotenoids [9]. Polyphenols also have anti-inflammatory effects [10]. Increasing evidence has shown that polyphenols have a beneficial impact on the prevention of diseases associated with oxidative stress such as cancer, atherosclerosis, inflammation, and neurodegenerative diseases (e.g., Alzheimer's and Parkinson's) [11-14].

Alzheimer's disease (AD) is the major cause of dementia in humans, but no drug or treatment for the disease has been discovered so far. The disease is characterized by extracellular plaques of amyloid beta $(\mathrm{A} \beta)$ fibrils, intracellular neurofibrillary tangles of hyperphosphorylated and misfolded tau protein, vascular damage resulting from extensive plaque deposition, and the loss of neuronal cells and synapses [15]. According to the most influential hypothesis about the pathogenesis of AD, amyloid cascade, that is, the deposition of $A \beta$ peptide into plaques in brain tissue, is the causative agent of the disease [16]. Generated from the cleavage of a single-pass transmembrane protein, amyloid precursor protein (APP), $A \beta$ is an amphiphilic and partly folded molecule, thus being prone to self-aggregate and produce intermediate oligomers or protofibrils, and finally insoluble fibrils [17]. A $\beta$ impairs the activity of some membrane transporters, increases cellular oxidative stress, and causes neuroinflammation, thereby inducing extensive synapse dysfunction and neuron loss [17,18]. It has been reported that the neurotoxicity of $A \beta$ strongly depends on their aggregated state, in which oligomers and protofibrils are more toxic than soluble monomers and mature fibrils. With a high ability to interact with cell membranes, $A \beta$ oligomers and protofibrils form pores, disrupt membrane receptors, alter membrane properties, and induce the accumulation of intraneuronal $A \beta$ [19]. However, the exact mechanisms remain unknown. Thus, the unravelling of factors that affect $A \beta$ aggregation is important toward the prevention and treatment of AD.

The inhibitory or modulating effects on $A \beta$ aggregation have been found in 44 polyphenolic compounds [20]. Ono et al. (2003) reported that wine-related phenols (myrectin, morin, quercetin, kaempferol (+)-catechin and (-)-epicatechin) inhibited the formation of $A \beta$ fibrils from fresh $A \beta \_40$ and $A \beta \_42$ and destabilized preformed $A \beta$ fibrils in dose-dependent manner [21]. Similar effects of curcumin, rosmarinic acid, tannic acids, epigallocatechin gallate (EGCG), olive tree-extracted polyphenols, resveratrol, and other stilbenes were identified [22-26]. Furthermore, in vivo studies reported that some polyphenols such as EGCG, resveratrol, and curcumin decreased amyloid levels and plaque formation in mice brains [27-29]. The ability of polyphenols to prevent $A \beta$ polymerization was proposed to be mediated by their direct interaction with $\mathrm{A} \beta$ or by their binding to ions, which facilitate $A \beta$ aggregation [20]. Despite the numerous studies demonstrating the beneficial impact of some polyphenols in A $\beta$ self-assembly, the anti-amyloidogenic properties of other various polyphenolic compounds are not fully understood. More efforts are needed in order to elucidate structure-function relationship(s).

In this study, we aimed to investigate the effect of polyphenols on the self-assembly of $A \beta$ peptide, in particular, how this relates to structure. Two classes of polyphenols, namely, trans-stilbenes and flavonoids, were used to assess whether the gallate side chains in flavonoids or the glucoside moiety in stilbenes could provide essential insights about the interactions of structurally different polyphenols with amyloid beta peptide. For stilbenes, we chose resveratrol, the main ingredient of polyphenols in wine, and its major derivative piceid (Figure S1A,B). For flavonoids, we selected gallocatechin gallate (GCG) and theaflavin (TF) (Figure S1C,D). TF is one of the main phenolic components found in fermented black tea, whereas GCG is an epimer of EGCG, a major polyphenol in green tea and a well-known inhibitor of $A \beta$ aggregation, but it is more stable and bioactive [30]. Resveratrol has been reported extensively in literature, thus providing us a good reference point for our current study $[26,28]$. As far as we are aware, there is no study about the effect of GCG on A $\beta$ aggregation, while piceid and $\mathrm{TF}$ and have been reported only once [26,31]. Having structures that are closely related (between class comparison) and related at the functional group level (within class comparison) (Figure S1) enabled us 
to draw a structure-dependent relationship between the polyphenols and their interaction with amyloid beta. The findings of this study may help in advancing our understanding of the physicochemical mechanism involved in the bioactivity of polyphenols as potential neuro-protective agents.

\section{Materials and Methods}

\subsection{Materials}

Polyphenols of $>97 \%$ purity were purchased: TF from Wako Pure Chemical (Tokyo, Japan); piceid from LKT Laboratories (Tokyo, Japan); resveratrol, GCG from Sigma-Aldrich (St. Louis, MO, USA). All other reagents were purchased from Wako Pure Chemical (Tokyo, Japan) and were of analytical grade. Deionized water obtained from Millipore Milli-Q purification system (Millipore, Bedford, MA, USA) was used for reagent preparation and for cleaning of glassware.

\subsection{Preparation of Amyloid Beta}

$\mathrm{A} \beta$ peptides were prepared in $0.02 \%$ ammonia solution at $0.2 \mathrm{mM}$ and stored $\mathrm{at}-80^{\circ} \mathrm{C}$. Just before analysis, and after equilibration at room temperature ( $\left.\mathrm{RT}=24 \pm 1^{\circ} \mathrm{C}\right)$, the peptides $(80 \mu \mathrm{M})$ were diluted in $20 \mathrm{mM}$ Tris-HCl buffer solution (TBS) pH 7.4, and allowed to spontaneously aggregate in TBS at $37 \pm 1{ }^{\circ} \mathrm{C}$ for a specific period of time. All analyses were carried out at RT. Prior to binding assays, the $A \beta$ solution was prepared by dilution with TBS to a final concentration of $20 \mu \mathrm{M}$. Each measurement was performed on a freshly extracted aliquot of the protein sample kept at $37 \pm 1{ }^{\circ} \mathrm{C}$ for aggregation.

\subsection{Preparation of Polyphenol Solutions}

GCG and piceid were prepared by dissolving in Milli-Q. TF and resveratrol were prepared by dissolving in aqueous methanol. All stock solutions were made at the concentration of $1 \mathrm{mM}$ and stored at $-25^{\circ} \mathrm{C}$. When they were used for experiments, methanol was diluted 10 times with Milli $Q$ water in all of them. The final working solution was $100 \mu \mathrm{M}$.

\subsection{Incubation of Amyloid Beta with and without Polyphenols}

Samples of $\mathrm{A} \beta(80 \mu \mathrm{M})$ in TBS without and with polyphenols under different concentrations were incubated in an incubator at $37 \pm 1^{\circ} \mathrm{C}$. The samples were in quiescence during the incubation, except for a few seconds of mixing prior to the measurement. In order to assess the behavior of the peptide through time, we took measurements after $0 \mathrm{~h}, 12 \mathrm{~h}$, and $24 \mathrm{~h}$ incubation. First, we investigated the behavior of $A \beta$ incubated without polyphenols. Then, we considered various incubation times of $A \beta$ with individual polyphenols, separately. We also checked each polyphenol's behavior by incubating each of them separately with and without ThT. Specifically, we wanted to see if they would interact with ThT assay and cause interference.

\subsection{Amyloid Beta Aggregation Kinetics Using Thioflavin-T Assay}

Kinetics of $A \beta$ fibril formation was analyzed using thioflavin $\mathrm{T}$ (ThT) fluorescence assay [32]. Fluorescence intensity was monitored at an excitation wavelength of $450 \mathrm{~nm}$ and an emission wavelength of $483 \mathrm{~nm}$ using a spectrofluorophotometer (FP-6500, Jasco, Tokyo, Japan). The buffer background was subtracted. The signals were collected at $0 \mathrm{~h}, 12 \mathrm{~h}$, and $24 \mathrm{~h}$ incubation.

The inhibition percentages on $\mathrm{A} \beta$ aggregation ( $I \%$ ) were calculated as follows [26]:

$$
I \%=\frac{I_{\text {control }}-I}{I_{\text {control }}} \times 100
$$

The data are expressed as means \pm standard deviation (SD) for three independent experiments. Comparisons between $\mathrm{Cu}$, polyphenols, and the control were performed using ANOVA analysis. 


\subsection{Morphological Observation Using Atomic Force Microscopy}

Atomic Force Microscopy (AFM) was used to image and characterize the conformation of A $\beta \_42$ aggregates derived from the incubation of $A \beta \_42$ alone or with lipid vesicles. In order to prepare the AFM samples, a $5 \mu \mathrm{M}$ portion of $A \beta \_42$ solution was uniformly spread and immobilized in a mica plate (Furuuchi Chemical Co.; Shinagawa, Tokyo, Japan). Then, the mica was washed three times with $50 \mu \mathrm{L}$ of deionized water to exclude Tris buffer molecules and was dried under vacuum conditions. The sample was measured by AFM (SPA400-SPI 3800, Seiko Instruments Inc., Chiba, Japan) equipped with a calibrated $20 \mathrm{~lm}$ xy-scan, a $10 \mathrm{lmz}$-scan range PZT-scanner, and a scanning silicon nitride tip (SI-DF3, spring constant $=1.6 \mathrm{~N} / \mathrm{m}$, frequency resonance $=28 \mathrm{kHz}$, Seiko Instruments Inc.) in a dynamic force mode (DFM). All AFM operations were performed in an automated moisture control box with $30-40 \%$ humidity at room temperature. The length and height of $A \beta \_42$ aggregates were analyzed using ImageJ and SPI software, respectively [33].

\subsection{Characterization of Redox Activity of Polyphenols}

The electrochemical voltammetric technique was used to characterize the redox activity of polyphenols [34]. Experiments were conducted with $A \beta(80 \mu \mathrm{M})$ incubated alone, polyphenols $(80 \mu \mathrm{M})$ incubated alone, and $A \beta$ incubated with polyphenols (molar ratio $A \beta$ :polyphenols 1:1). All were incubated for $0 \mathrm{~h}, 12 \mathrm{~h}$, and $24 \mathrm{~h}$ at $37 \pm 2{ }^{\circ} \mathrm{C}$. Differential pulse voltammetry (DPV) analyses were performed with a BDTminiSTAT100 Potentiostat (Bio-Device Technology, Inc., Ishikawa, Japan) in connection with its KME-UsbStat software. The electrode system consisted of triangular disposable electrochemical printed (DEP) chips (Bio-Device Technology, Inc.; Ishikawa, Japan) consisting of a carbon working electrode, a platinum counter electrode, and a $\mathrm{Ag} / \mathrm{AgCl}$ reference electrode, connected to the mini analyzer with a measuring electrode cable. DEP chips were rinsed with PBS before each individual analysis. A volume of analyte $(20 \mu \mathrm{L})$ was carefully dropped onto the DEP chips, and analyzed immediately. DPV parameters were as follows: scan range $-0.3 \mathrm{~V}$ to $1.2 \mathrm{~V}$; step potential $5 \mathrm{mV}$; modulation amplitude $25 \mathrm{mV}$; and scan rate $50 \mathrm{mV} / \mathrm{s}$.

\section{Results and Discussion}

\subsection{Polyphenol-Modulated Amyloid Beta Aggregation}

First, we assessed how polyphenols influence $A \beta \_42$ self-assembly using thioflavin $T$ (ThT) assay, a common analytical method for detecting the degree of amyloid fibrillation [35]. When ThT binds to the $\beta$-sheet of $A \beta \_42$, it shows enhanced fluorescence emission at $483 \mathrm{~nm}$ wavelength, instead of at $445 \mathrm{~nm}$ wavelength as free ThT [36]. Our previous study reported that $80 \mu \mathrm{M} \mathrm{A} \beta$ monomers in Tris- $\mathrm{HCl}$ buffer ( $\mathrm{pH} 7.4$ ) incubated at $37^{\circ} \mathrm{C}$ for $12 \mathrm{~h}$ and $24 \mathrm{~h}$ spontaneously aggregated into prefibrils and fibrils, respectively [37]. Thus, we herein incubated $80 \mu \mathrm{M} A \beta$ monomers in Tris- $\mathrm{HCl}$ buffer supplemented with and without polyphenols (1:1 molar ratios) for $12 \mathrm{~h}$ and $24 \mathrm{~h}$, then analyzed the extent of amyloid fibrillation at various incubation periods based on the detected ThT fluorescent intensity at $483 \mathrm{~nm}$ wavelength. We also measured the aggregation extent of $A \beta \_42$ incubated with $\mathrm{Cu}^{2+}$ ions, reported to inhibit $\mathrm{A} \beta$ fibrillation [38,39], as a positive control.

The results show that the aggregation kinetics of $A \beta \_42$ increased steadily over the duration of the experiment (Figure 1A, insert). This was consistent with our previous studies, in which we reported that the peptide's aggregation behavior followed a typically sigmoidal curve [33], which starts to reach the equilibrium after $24 \mathrm{~h}$ incubation. In agreement with previous studies, $\mathrm{Cu}^{2+}$ hindered $\mathrm{A} \beta \_42$ fibril formation, at about $70 \%$ and $80 \%$ after $12 \mathrm{~h}$ and $24 \mathrm{~h}$ incubation periods, respectively (Figure 1 ). The presence of polyphenols induced significant changes in the aggregation kinetics of $A \beta \_42$. The peptide incubated with resveratrol showed a remarkable increase in ThT fluorescence compared to the control, indicating increased $A \beta$ aggregation, at $12 \mathrm{~h}$ incubation. However, the fluorescence intensity decreased significantly at $24 \mathrm{~h}$ incubation (Figure 1A). The results indicate that resveratrol reduced the aggregation extent of $A \beta$ in general, but it accelerated the initial aggregation of the peptide 
from monomeric to fibrillar species. The presence of piceid, a glucoside derivative of resveratrol, caused very little change in the aggregation of $A \beta$ for the first $12 \mathrm{~h}$. Afterwards, the aggregation kinetics was slowed down noticeably after a $24 \mathrm{~h}$ incubation period as shown by ThT fluorescence intensity (Figure 1A, insert). Contrary to the results with stilbenes, when $A \beta$ was incubated with two flavonoids (GCG and TF), the fluorescence intensity of ThT was hardly observed, implying that the aggregation of $\mathrm{A} \beta$ was completely inhibited in the presence of the two flavonoids (Figure 1).
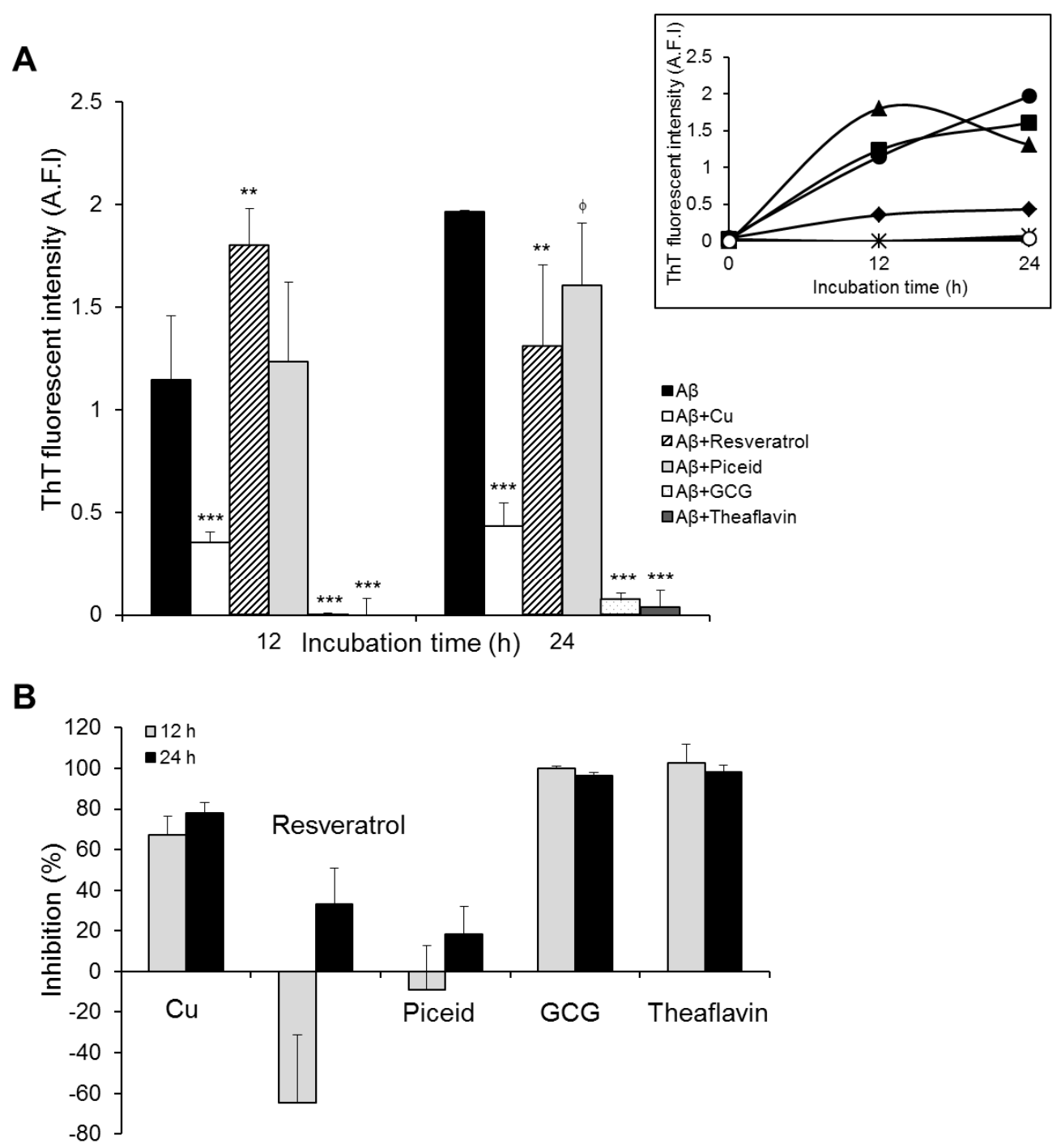

Figure 1. Inhibitory activity of copper ion and polyphenols on $A \beta \_42$ aggregation. (A) ThT fluorescence intensity was measured after incubation of $A \beta$ without and with $\mathrm{Cu}$ and polyphenols for a period of $0 \mathrm{~h}$, $12 \mathrm{~h}$, and $24 \mathrm{~h}$ (main figure). Time course curves of $\mathrm{A} \beta \_42$ aggregation in the absence (black, circle) and the presence of $\mathrm{Cu}$ (black, diamond), resveratrol (black, triangle), piceid (black, square), gallocatechin gallate (GCG) (black, star), and theaflavin (TF) (white, circle) (inserted figure). (B) Inhibition percentages of polyphenols on $\mathrm{A} \beta$ aggregation. All values represent means $\pm \operatorname{SD}(n=3) ;{ }^{* *}, p<0.01 ;{ }^{* * *}, p<0.001$; $\phi, p<0.1$.

These results are very interesting. They suggest a contradictory mechanism for the polyphenols' inhibitory effects on $A \beta$ aggregation. The two flavonoids could inhibit $A \beta$ aggregation close to $100 \%$. The presence of stilbenes (resveratrol and piceid) could also inhibit $A \beta$ aggregation, but to a much lesser extent (Figure 1B). Intriguingly, resveratrol exhibited an accelerating effect on the formation of $A \beta$ fibrils before the inhibitory activity was detected (Figure 1B). With respect to the comparison within class, hardly any difference in anti-A $\beta$ aggregation property between the two flavonoids was 
observed, whereas resveratrol was slightly more anti-aggregative against the peptide than piceid but only after $24 \mathrm{~h}$ incubation. In order to understand more about the differences in the modulation of $A \beta$ self-assembly by these two classes of polyphenols, we observed nanoscale morphological changes in $A \beta \_42$ aggregates resulting from the $24 \mathrm{~h}$ incubation of the peptide with the polyphenols using AFM.

\subsection{Morphologies of Amyloid Beta Self-Assembly in the Presence of Polyphenols}

AFM was used to image morphologies of $A \beta \_42$ formed in the presence and absence of polyphenols following incubation at $37^{\circ} \mathrm{C}$ for $24 \mathrm{~h}$. As can be seen in Figure 2, stilbenes and flavonoids induced $A \beta \_42$ to aggregate into two different morphological species. When the peptide was incubated with stilbenes for $24 \mathrm{~h}$, most of the observed aggregates had long and branched nanostructures, similar to aggregated species of the peptide by itself (Figure 2B-D). The length and height of peptide aggregates were largely in the range from 150 to $400 \mathrm{~nm}$ and from 2 to $6 \mathrm{~nm}$, respectively (Figure S2B,C). This clearly showed that $A \beta \_42$ was mostly fibrillar. It is interesting to note that $A \beta$ fibrils following incubation with resveratrol were longer and more branched than those from incubation with piceid, implying a faster fibrillation of $\mathrm{A} \beta$ under the effect of resveratrol (Figure 2C). Conversely, the peptide did not form fibrillar aggregates in the presence of flavonoids. Only small, spherical, and unstructured particles were imaged (Figure 2E,F). Nearly $85 \%$ of these particles were 2-6 nm high and they were shorter than $100 \mathrm{~nm}$ (Figure S2D,E). The ability of EGCG, the epimer of GCG, and TF to promote the assembly of $A \beta$ monomers into nontoxic, spherical, and amorphous aggregates was reported previously [31,40]. Thus, we imagine that the two flavonoids inhibited the fibril formation and induced the formation of non-toxic aggregates.
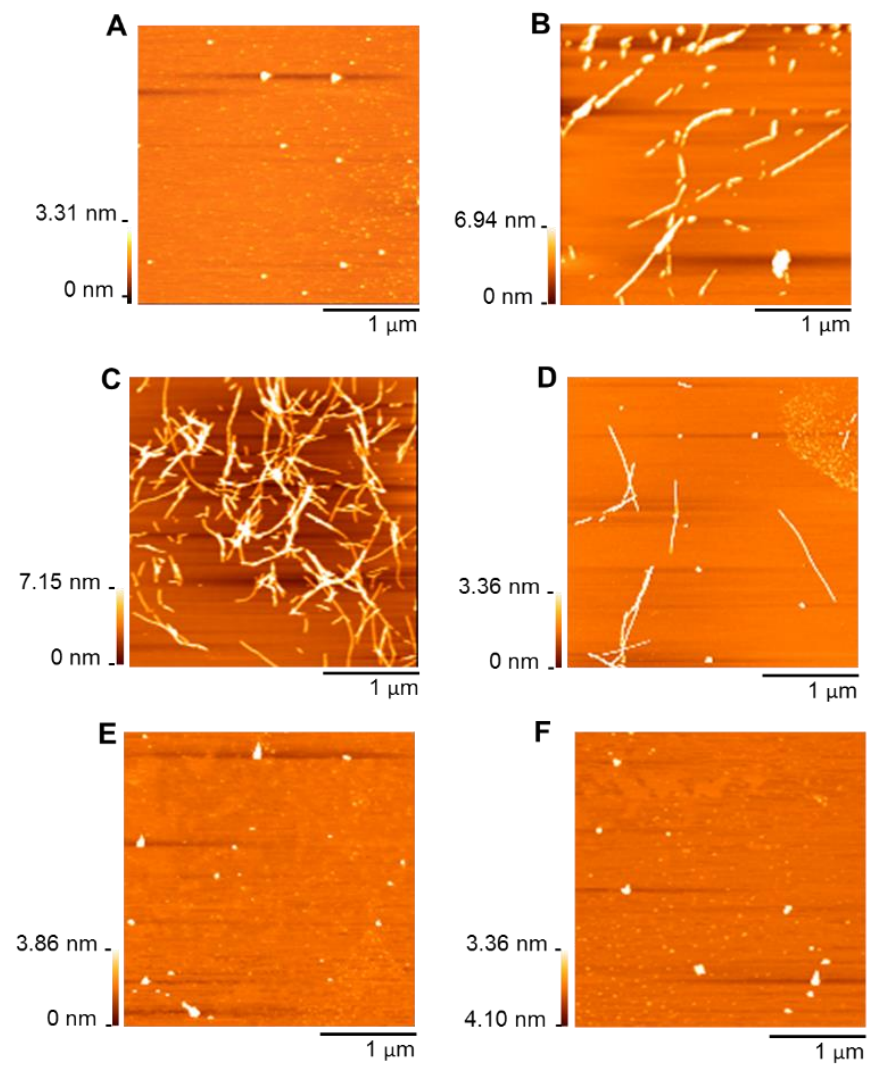

Figure 2. Morphology of (A) $A \beta \_42$ monomers and $A \beta \_42$ aggregates obtained after incubation (B) without polyphenols and with (C) resveratrol, (D) piceid, (E) GCG, and (F) TF for $24 \mathrm{~h}$. The samples were analyzed using atomic force microscopy (AFM) in a dynamic force mode. All AFM operations were performed in an automated moisture control box with 30-40\% humidity at room temperature. The length and height of $A \beta \_42$ aggregates were analyzed using ImageJ and SPI software, respectively. 
The results of the AFM experiment were consistent with our ThT assay above, indicating the strikingly different effects of two polyphenol classes, stilbenes and flavonoids, on the aggregation of $A \beta \_42$. While the stilbenes decreased $A \beta$ aggregation after accelerating the peptide to assemble into the less toxic fibrillary species, the latter completely hindered the fibrillogenesis. In order to investigate further the relationship between the structure of these polyphenols and their impact on $A \beta$ aggregation, we then conducted an electrochemical analysis. We studied the interaction between the polyphenols and $A \beta \_42$ by measuring the redox activity of polyphenols in the presence and absence of $A \beta$ after three different incubation periods $(0 \mathrm{~h}, 12 \mathrm{~h}$, and $24 \mathrm{~h})$. Since the antioxidant activity of polyphenols is attributed to their chemical structure, in particular the presence of aromatic $\mathrm{OH}$ groups, their location relative to each other, the oxidation state of the C-ring, and the overall number of $\mathrm{OH}$ groups present, the information could be important to understand the role of polyphenol structure in their activity on $A \beta$ aggregation [6].

\subsection{Redox Activity of Polyphenols in the Presence of Amyloid Beta}

The electrochemical properties of GCG, TF, resveratrol, and piceid in PBS (50 mM, pH 7.4) and $\mathrm{A} \beta \_42$ in Tris buffer in $0 \mathrm{~h}$ (the control), $12 \mathrm{~h}$, and $24 \mathrm{~h}$ incubation at $37^{\circ} \mathrm{C}$ were analyzed using differential pulse voltammetry (DPV) at carbon disposal electrochemical screen-printed (DEP) chips. Typical voltammograms of TF and piceid in the presence and absence of $A B$ are shown in Figure 3.

The number and position of peak potentials are shown in Table 1. A $\beta \_42$ monomer displayed one peak potential at $0.71 \mathrm{~V}$, which is attributed to the oxidation of tyrosine residue [41]. However, the peak potential completely disappeared after $12 \mathrm{~h}$ and $24 \mathrm{~h}$ incubation of $\mathrm{A} \beta \_42$ due to the assembly of the monomer to form aggregated species, in agreement with our previous study [35]. All polyphenols had at least two oxidation peak potentials, but the peak number and position varied significantly according to the class and also the particular polyphenol.

Table 1. Peak potentials of polyphenol $(80 \mu \mathrm{M})$ solutions incubated at $37^{\circ} \mathrm{C}$ for $0 \mathrm{~h}, 12 \mathrm{~h}$, and $24 \mathrm{~h}$.

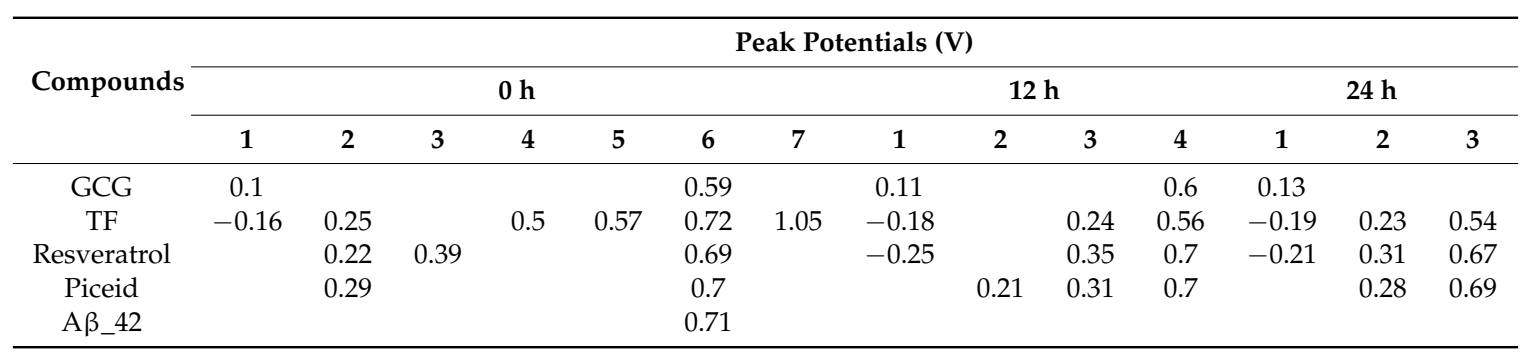

Resveratrol and piceid showed the first peak potentials at $0.22 \mathrm{~V}$ and $0.29 \mathrm{~V}$, respectively, corresponding to the oxidation of hydroxyl group on B-ring. The second potential of the two stilbenes appearing around $0.69 \mathrm{~V}$ was characterized as corresponding to the resorcinol group of resveratrol and hydroxyl groups of piceid on the A-ring. At similar conditions to our reports, we previously reported oxidation of the resorcinol group at $\sim 0.71 \mathrm{~V}$ at the glassy carbon electrode [34]. In addition to the characterized peak potentials, one more oxidation peak potential was observed for resveratrol at $0.39 \mathrm{~V}$. This could be explained by the further oxidation of resorcinol oxidation product(s) [42]. After $12 \mathrm{~h}$ and $24 \mathrm{~h}$ incubation at $37^{\circ} \mathrm{C}$, both stilbenes showed small changes in peak potentials. Resveratrol displayed one new peak potential at $-0.25 \mathrm{~V}$, whereas the peak at $0.39 \mathrm{~V}$ had disappeared. After $12 \mathrm{~h}$ incubation, piceid had a third peak potential at $0.21 \mathrm{~V}$, which may correspond to the oxidation of the glucoside moiety. However, this peak potential completely disappeared after incubation for $24 \mathrm{~h}$. As far as we are aware, electroanalysis of piceid has not been previously studied. 
A

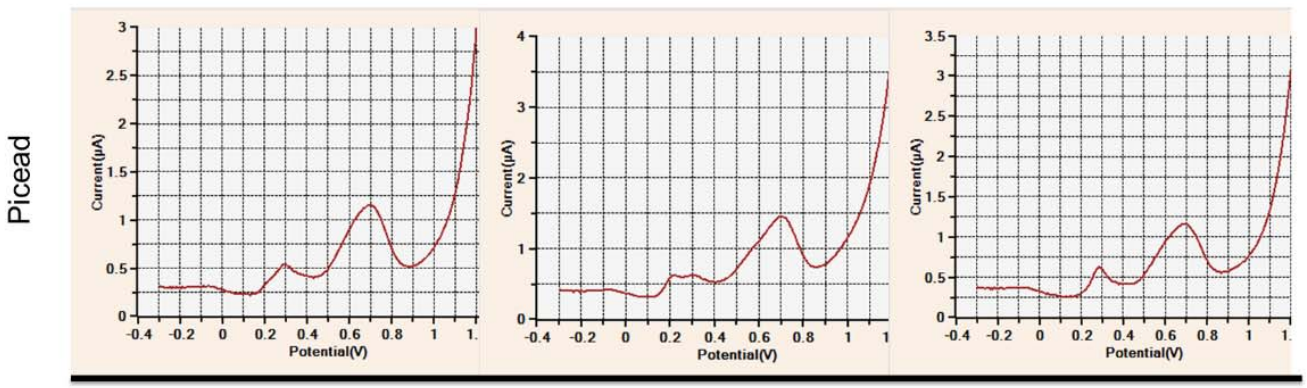

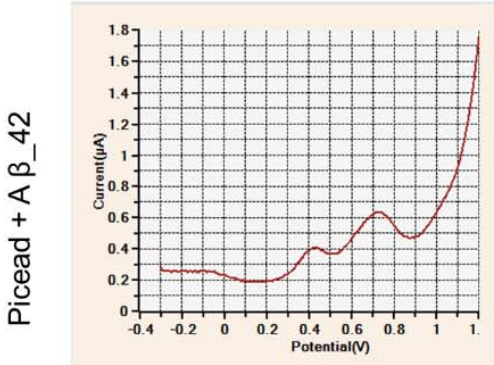

$\mathrm{Oh}$

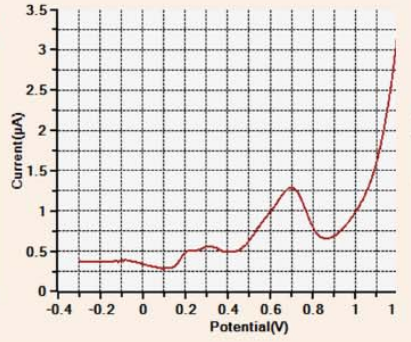

$12 \mathrm{~h}$

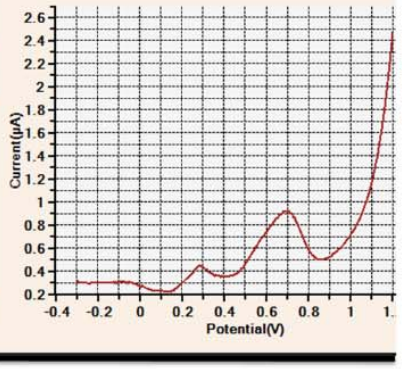

$24 \mathrm{~h}$

\section{Incubation Period}

B

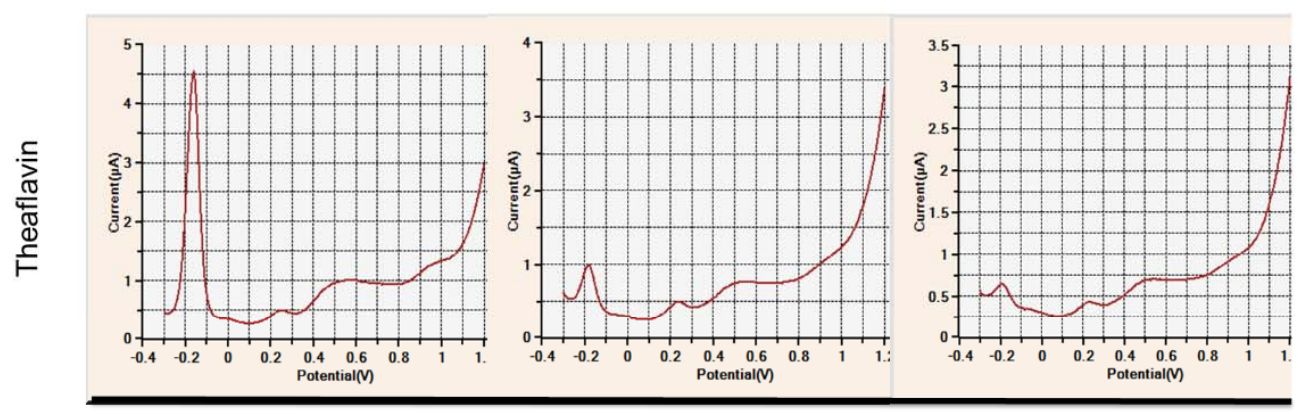

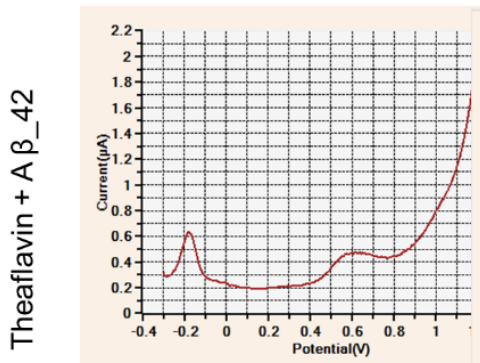

$\mathrm{Oh}$

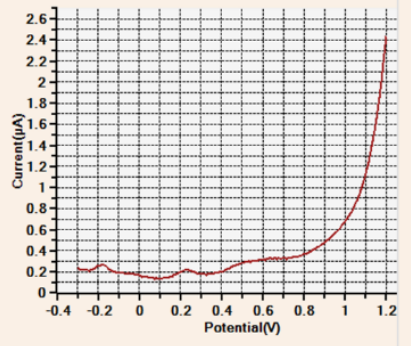

$12 \mathrm{~h}$

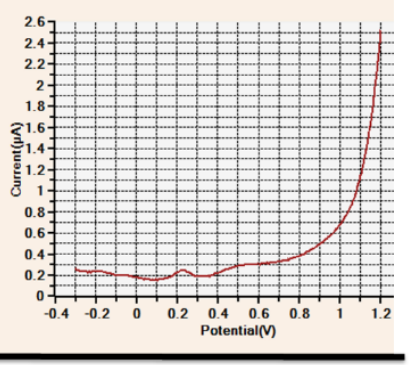

$24 \mathrm{~h}$

\section{Incubation Period}

Figure 3. Typical voltammograms of (A) picead (i) and piceid plus $A \beta \_42$ (ii); (B) theaflavin (TF) (i) and TF plus A $\beta \_42$ (ii) analyzed using differential pulse voltammetry (DPV) at carbon disposal electrochemical screen-printed (DEP) chips. DPV parameters were as follows: scan range $-0.3 \mathrm{~V}$ to $1.2 \mathrm{~V}$; step potential $5 \mathrm{mV}$; modulation amplitude $25 \mathrm{mV}$; and scan rate $50 \mathrm{mV} / \mathrm{s}$.

In case of the flavonoids, GCG had two oxidation peak potentials at 0.1 and $0.59 \mathrm{~V}$, corresponding to the oxidation of pyrogallol/galloyl groups in B/D-rings and the resorcinol group in the A-ring [34]. The oxidation of $\mathrm{TF}$, on the other hand, was more complicated to interpret. There were six peak 
potentials. Based on the similarity of TF with catechin, we assume that the $-0.16 \mathrm{~V}, 0.5 \mathrm{~V}$, and $0.57 \mathrm{~V}$ oxidation potentials corresponded to its catechol groups in the fused seven-membered benzotropolone ring and $\mathrm{C}-3 \mathrm{OH}$ groups of two C-rings, whereas the $0.72 \mathrm{~V}$ and $1.05 \mathrm{~V}$ peak potentials could correspond to the oxidation of resorcinol groups on the A-rings [34]. The peak potential at $0.25 \mathrm{~V}$ might correspond to the oxidation of the $\mathrm{OH}$ group in conjunction with the oxo group on the benzotropolone ring. Wu et al. suggested that the benzotropolone moiety might play an important role in the antioxidation of TF [43], and the oxidation of the C $-5 \mathrm{OH}$ group in conjunction with the C-4 oxo group was reported previously [34]. After $12 \mathrm{~h}$ and $24 \mathrm{~h}$ incubations, of the six oxidation potentials, only two could be observed. Meanwhile, the second oxidation peak potential for GCG could not be observed after incubation for $24 \mathrm{~h}$. The changes in peak potentials of polyphenols after incubation might be due to auto-oxidation.

There were varying changes in the peak potentials of polyphenols in the presence of $A \beta \_42$ (Table 2). Resveratrol and piceid still displayed two peak potentials, corresponding to the oxidation of the hydroxyl group on C- $4^{\prime}$ and resorcinol groups. However, the first oxidation occurred at much higher potentials, namely, $0.45 \mathrm{~V}$ for resveratrol and $0.43 \mathrm{~V}$ for piceid, whereas the current detected for the second significantly decreased (by nearly 50\% of the control, Tables S1 and S2). This indicates a decrease in the availability of functional groups to be oxidized. The incubation of stilbenes with $\mathrm{A} \beta \_42$ for $12 \mathrm{~h}$ led to the loss of the first peak potentials and the appearance of new peak potentials at $0.34 \mathrm{~V}$ and $0.31 \mathrm{~V}$ for resveratrol and piceid, respectively. This may suggest that the $\mathrm{OH}^{-}$group on C-4' had either been completely complexed with $\mathrm{A} \beta \_42$ and that the complex was redox-active at new potentials, or that the interaction with $\mathrm{A} \beta \_42$ had changed the conformation structure of the stilbenes, making the $\mathrm{OH}^{-}$group on $\mathrm{C}-4^{\prime}$ more difficult to oxidize. Meanwhile, the second peak potential of both stilbenes shifted to slightly higher potentials. Piceid showed an additional oxidation potential at $0.22 \mathrm{~V}$, which might reflect the oxidation of the glucoside group. When the incubation time increased to $24 \mathrm{~h}$, the second peak potential of resveratrol could not be seen, while the oxidation potential from the glucoside moiety in piceid remained unchanged. This could imply that glucoside moiety might inhibit (perhaps due to steric hindrance) the ability of the hydroxyl group on C-5 to interact with the peptide.

Table 2. Peak potentials of polyphenol $(80 \mu \mathrm{M})$ solutions incubated with $\mathrm{A} \beta \_42$ at $37^{\circ} \mathrm{C}$ for $0 \mathrm{~h}, 12 \mathrm{~h}$, and $24 \mathrm{~h}$.

\begin{tabular}{|c|c|c|c|c|c|c|c|c|c|c|c|c|}
\hline \multirow{3}{*}{ Polyphenols } & \multicolumn{12}{|c|}{ Potentials (V) } \\
\hline & \multicolumn{4}{|c|}{$0 \mathrm{~h}$} & \multicolumn{4}{|c|}{$12 \mathrm{~h}$} & \multicolumn{4}{|c|}{$24 \mathrm{~h}$} \\
\hline & 1 & 2 & 3 & 4 & 1 & 2 & 3 & 4 & 1 & 2 & 3 & 4 \\
\hline GCG & 0.14 & & 0.67 & & 0.11 & & & & 0.11 & & & \\
\hline $\mathrm{TF}$ & -0.18 & & 0.63 & 1.07 & -0.18 & & 0.23 & 0.66 & & 0.23 & 0.49 & \\
\hline Resveratrol & & 0.45 & 0.66 & & & & 0.34 & 0.71 & & & 0.35 & \\
\hline Piceid & & 0.43 & 0.73 & & & 0.22 & 0.31 & 0.7 & & & 0.27 & 0.69 \\
\hline
\end{tabular}

The redox activity of GCG in the presence of $A \beta \_42$ showed a small shift in the oxidation potentials to the right, and a remarkable decrease in anodic current was detected (approximately $85 \%$ for the first and 35\% for the second potential, Tables S1 and S2). After $12 \mathrm{~h}$ and $24 \mathrm{~h}$ incubation, the peak potential at $0.67 \mathrm{~V}$ was not observable, suggesting a possible complete complexation of resorcinol moiety and partial interaction of pyrogallol moiety/gallate side chain with A $\beta \_42$. In the case of TF, this flavonoid showed the biggest change among the four polyphenols upon the presence of $A \beta \_42$. It lost three oxidation potentials at $0.25,0.5$ and $0.57 \mathrm{~V}$, corresponding to the oxidation of the $\mathrm{OH}$ group in conjunction with the oxo group on the benzotropolone ring and C-3 OH groups on two C-rings. The current detected of the first peak potentials, which reflect the oxidation of catechol, considerably dropped by approximately $85 \%$, whereas that of the two remaining oxidation peaks corresponding to two resorcinol groups only decreased slightly. The oxidation potential of one resorcinol group disappeared and was replaced with a new potential at $0.23 \mathrm{~V}$ after $12 \mathrm{~h}$ incubation of TF with A $\beta \_42$. When the incubation time increased to $24 \mathrm{~h}$, TF lost the two last peak potentials and displayed one 
more new potential at $0.49 \mathrm{~V}$. These results suggest that all redox-active "natural" functional groups of TF may have been directly involved in the interaction with A $\beta \_42$ and that the interacted products of at least one of the groups were oxidized at 0.23 and $0.49 \mathrm{~V}$.

\subsection{Modulation of Amyloid Beta Aggregation by Stilbenes and Flavonoids}

The results of ThT assays and AFM experiments showed that both stilbenes and flavonoids could affect amyloid beta aggregation, but in different ways. Flavonoids (GCG and TF) could completely prevent fibril formation of $A \beta \_42$ and remodel the peptide to assemble into non-toxic, unstructured aggregates. In comparison, stilbenes displayed a lesser anti-A $\beta$ aggregation activity (Figures 1 and 2). Previous studies have reported on the inhibitory effect of TF [31], an epi form of GCG [40], resveratrol [26,28] and piceid [26] on $A \beta$ fibrillogenesis. However, as far as we are aware, our study is the first to report on flavonoids exhibiting a higher anti-A $\beta$ aggregation potential than stilbenes. We investigated the structure-activity relationship of polyphenols and their effects on $A \beta$ aggregation aside from their antioxidant properties. The main structural differences between the two classes of polyphenols are (i) the presence of an aromatic ring instead of an olefin bond for flavonoids and (ii) the presence of a gallate group on C-3 or the dimerization at B-rings producing a fused seven-membered benzotropolone ring and additional A- and C-rings for the flavonoids. This means that flavonoids possess more aromatic rings and hydroxyl groups than stilbenes, which most likely confers on them their higher anti-aggregative activity against $A \beta$ compared to the latter. Numerous studies have showed that anti-aggregation compounds can exert their effects by forming covalent and non-covalent interactions such as $\pi-\pi$ stacking interactions, hydrogen bonding, or charge-charge interactions between an inhibitor and the backbone of side-chain residues of the target protein. The presence of rings in the chemical structure of polyphenols enables them to form covalent interactions with hydrophobic amino-acid residues (Tyr, Phe), whereas hydroxyl groups bind to hydrophilic amino-acid residues (His6, Ser8, Tyr10, His14, Lys16) of A $\beta$ [20,44,45]. Our electrochemistry data indicated a higher ability of flavonoids to interact with the peptide compared to stilbenes. Pyrogallol moiety/gallate side chain of GCG and catechol of TF are the most potent group binding to A $\beta$, as demonstrated by a dramatic plunge in the current detected for their oxidation potentials (about $85 \%$ upon $0 \mathrm{~h}$ incubation, Tables S1 and S2). TF lost three oxidation potentials at $0 \mathrm{~h}$ incubation with $\mathrm{A} \beta$, and the potentials of the other oxidizable groups could not be observed when the incubation time increased, suggesting that all functional groups of the flavonoid interacted completely with the peptide (Table 2). In addition to a high number of aromatic rings and hydroxyl groups, the presence of phenolic rings in all sides of GCG and TF molecules (Figure S1C,D) is thought to form a "hydroxyl edge" on each side of polyphonic compounds that might improve binding to polypeptide chains [44]. On the other hand, the opening of the benzyl ring forms a linear shape in stilbenes with two hydrophilic ends and the central hydrophobic region (Figure S1A,B), likely reducing the stilbenes' interactive ability with A $\beta$, relative to flavonoids.

Of additional interest is the faster $\mathrm{A} \beta$ aggregation in the presence of resveratrol, during the first $12 \mathrm{~h}$ incubation, followed by a significant decrease of fibrillation extent at $24 \mathrm{~h}$ incubation compared to the control (Figure 1). This suggests the ability of resveratrol to induce a remarkably faster formation of $\mathrm{A} \beta$ fibrils during the first $12 \mathrm{~h}$ incubation, before its decreasing effect on $\mathrm{A} \beta$ aggregation was detected. Although much has been studied using resveratrol, the anti-aggregative property of this polyphenol against $A \beta$ remains complicated to interpret. Most of the studies reported that resveratrol is able to inhibit A $\beta$ aggregation. However, Feng et al. (2009) demonstrated that the phenol could not prevent oligomer formation although this group detected its positive effect on the polymerization of $\mathrm{A} \beta \_42$ monomers and the destabilization of $A \beta \_42$ fibrils [45]. According to Ladiwala et al. (2010), resveratrol selectively remodeled $A \beta$ soluble oligomers, fibrillary intermediates, and fibrils into non-toxic, high molecular weight, and unstructured aggregates, but it did not alter the aggregation behavior of freshly dissolved $A \beta$ monomer. The authors suggested that resveratrol may promote conversion of soluble oligomers into either fibrillary intermediates or fibrils, and then remodel a common $\mathrm{A} \beta$ conformation 
into large, unstructured aggregates [46]. Based on previous studies and our results, we propose that resveratrol affects $A \beta$ aggregation through two main steps: (i) accelerate a fast conversion of $A \beta$ monomers into fibrillary species, which are less toxic and elicit more the phenol to remodel their structure [46]; (ii) remodel the structure of $A \beta$ fibrils into non-toxic, unstructured aggregates, resulting in the reduction of fibrillation extent. The mechanism by which the stilbene induces a fast formation of $A \beta$ fibrils before suppressing fibrillation is still unclear. One possibility involves the molecular conformation of resveratrol. It has been reported that the driving force bringing A $\beta$ nuclei together during aggregation process are non-native side-chain electrostatic and hydrophobic interactions [47]. Since resveratrol exhibits a central hydrophobic region and a small, linear shape (Figure S1A), it would be able to mediate the nucleation from $\mathrm{A} \beta$ monomers via hydrophobic interaction, thereby accelerating the formation of fibrils. When $A \beta$ fibrils are formed, aromatic staking, which is produced by the self-stacking of aromatic residues in amyloid core, might elicit resveratrol remodeling activity through interaction with the phenolic ring of the polyphenol, thus reducing the degree of aggregation [46].

Another important finding of our work is how changes in functional groups, within each class, have an impact on their effect on $A \beta$ aggregation. Specifically, we have semi-characterized the anti-amyloidogenic activities of polyphenols, at the functional group level. The presence of the gallate moiety on C-3 or the dimerization at B-rings producing a fused seven-membered benzotropolone ring and additional A- and C-rings (Figure S1C,D) appeared not to have influenced the anti-aggregative potency of flavonoids against $A \beta$. Although electrochemistry data indicated a higher ability of TF to interact with $A \beta \_42$ than GCG (Table 2), there was no difference in their effect on $A \beta$ aggregation, observed by ThT and AFM experiments (Figures 1 and 2E,F). This could imply that the electrochemical data may be more sensitive and provide more detailed information on the interaction between the peptide and the flavonoids. However, it is premature to draw that conclusion and further studies are required to confirm or refute it. The similarity between GCG's and TF's effects (AFM and ThT assay results) are in good agreement with Grelle et al.; who reported that TF and EGCG, an epi form of GCG, exhibit a similar propensity for $A \beta$ fibrillogenesis [31]. Another possibility would be that the differently anti-aggregative activities of the two flavonoids occurred in a shorter incubation time (less than $12 \mathrm{~h}$ ), so that they could not be detected in this study. Conversely, the addition of a sugar group on C-7 (Figure S1A,B) decreased the ability of piceid to inhibit amyloid beta aggregation, compared to resveratrol, which has a hydroxyl group instead, perhaps due to steric hindrance. The extent $A \beta$ fibrillation detected from $24 \mathrm{~h}$ incubation with piceid is slightly smaller than that from $24 \mathrm{~h}$ incubation with resveratrol, and the formation of $A \beta$ fibrils during $12 \mathrm{~h}$ incubation in the presence of piceid was relatively slow (Figures 1 and 2C,D). Similarly, using UV-Visible spectrophotometry, Rivière et al. (2007) showed resveratrol to have a slightly higher inhibitory activity on A $\beta$ aggregation in comparison with piceid [26]. Our electrochemistry data suggested that the change in the anti-A $\beta$ aggregation property of piceid might be attributed to the existence of glucoside on C-7 of the A-ring. Piceid displayed the peak potentials corresponding to the oxidation of glucoside and $\mathrm{OH}^{-}$groups on the A-ring during all incubation periods with $\mathrm{A} \beta$, whereas the oxidation potential of resorcinol group on the A-ring of resveratrol disappeared after $24 \mathrm{~h}$ incubation (Table 2). This suggests that the resorcinol group in resveratrol could interact completely with the peptide, whereas the interacting ability of the functional group on the A-ring of piceid was attenuated by the presence of glucoside.

\section{Conclusions}

In this paper, we conducted three experiments to investigate the modulating effect of polyphenols (flavonoids and stilbenes) on Alzheimer's A $\beta \_42$ aggregation. The results have shown the two polyphenol classes to inhibit $A \beta$ aggregation in different ways. On the one hand, flavonoids completely inhibited fibrillation of $A \beta$ monomers and induced the formation of spherical, unstructured aggregates. ThT assay results showed that both flavonoids exhibited a similar propensity for anti-A $\beta$ aggregation, regardless of some differently functional groups, in agreement with the AFM analysis. Electroanalysis of the interaction provided more light on the matter and suggested TF to have a higher 
interaction with the peptide than GCG. On the other hand, stilbenes were able to suppress $A \beta$ aggregation but to a significantly lesser extent. Interestingly, resveratrol, a common stilbene, accelerated the formation of $A \beta$ fibrils during the first $12 \mathrm{~h}$ incubation before decreasing the aggregation of the peptide. AFM images showed a huge mass of long and thin $A \beta$ fibrils formed in the presence of resveratrol. Although the morphology was the same in the presence of piceid, the fibrils were sparse. Electrochemical analyses indicate that resveratrol does have a higher degree of interaction with $\mathrm{A} \beta$ than does piceid. It is noteworthy that our results clearly demonstrate the importance of multi-analyses in order to understand better what could otherwise be interpreted as subtle differences, if not incorrectly. For example, the AFM images of $\mathrm{A} \beta$ fibrils at $24 \mathrm{~h}$ in the presence of resveratrol, on their own, could be misinforming.

We attributed the differences in bio-activities to the main structural differences between the two classes. The presence of an aromatic ring instead of an olefin bond and the substitution of the gallate group for C-3 hydroxyl or the dimerization at B-rings producing a fused seven-membered benzotropolone ring and additional A- and C-rings for the flavonoids result in a higher number of aromatic rings and hydroxyl groups in flavonoids than in stilbenes, thereby increasing the ability of the former class to interact with $A \beta$. In the comparison within classes, two flavonoids, GCG and theaflavin, appear to have a similar propensity for anti-A $\beta$ aggregation, although the electrochemical data show TF to be more interactive with A $\beta$ that GCG. However, the presence of glucoside on C-7 slightly reduced the inhibitory property of piceid, compared to resveratrol. These results are important to help unravel the potential of these polyphenols as $A \beta$ aggregation inhibitors, at a functional group level, which further opens the key to the tailored design of bioactive compounds as potential drug candidates.

Supplementary Materials: The following are available online at http:/ /www.mdpi.com/2072-6643/11/4/756/s1, Figure S1: Chemical structure of (A) resveratrol, (B) piceid, (C) gallocatechin gallate, and (D) theaflavin, Figure S2: (i) Length and (ii) height distribution of $A \beta \_42$ aggregates obtained from $24 \mathrm{~h}$ incubation (A) without polyphenols and (B) with resveratrol, (C) piceid, (D) gallocatechin gallate, and (E) theaflavin. A $\beta \_42$ was incubated at $80 \mu \mathrm{M}$ in Tris buffer $(20 \mathrm{mM}, \mathrm{pH} 7.4)$. The samples were using AFM in a dynamic force mode. All AFM operations were performed in an automated moisture control box with 30-40\% humidity at room temperature. The length and height of $A \beta \_42$ aggregates were analyzed using ImageJ and SPI software, respectively, Table S1: The current detected for peak potentials of polyphenol $(80 \mu \mathrm{M})$ solutions incubated at $37^{\circ} \mathrm{C}$ for $0 \mathrm{~h}, 12 \mathrm{~h}$, and $24 \mathrm{~h}$, Table S2: The current detected for peak potentials of polyphenol $(80 \mu \mathrm{M})$ solutions incubated with $\mathrm{A} \beta \_42$ at $37^{\circ} \mathrm{C}$ for $0 \mathrm{~h}$, $12 \mathrm{~h}$, and $24 \mathrm{~h}$.

Author Contributions: Conceptualization, M.C.V. and H.T.T.P.; methodology, M.C.V., K.S. and H.T.T.P.; formal analysis and investigation, K.S., A.F.A.-O., H.T.T.P. and M.C.V.; experiments, data curation, K.S., A.F.A.-O., Y.N., H.T.T.P.; writing—original draft preparation, H.T.T.P. and K.S.; writing—review and editing, H.T.T.P. and M.C.V.; supervision, Y.T. and M.C.V.

Funding: This research received no external funding

Acknowledgments: We are very grateful to Professor Masahiro Takagi of Japan Advanced Institute of Science and Technology (JAIST), for the use of laboratory equipment.

Conflicts of Interest: The authors declare no conflict of interest.

\section{References}

1. Pérez-Jiménez, J.; Neveu, V.; Vos, F.; Scalbert, A. Systematic analysis of the content of 502 Polyphenols in 452 foods and beverages: An application of the phenol-explorer database. J. Agric. Food Chem. 2010, 58, 4959-4969. [CrossRef] [PubMed]

2. Ignat, I.; Volf, I.; Popa, V.I. A critical review of methods for characterisation of polyphenolic compounds in fruits and vegetables. Food Chem. 2011, 126, 1821-1835. [CrossRef] [PubMed]

3. Pandey, K.B.; Rizvi, S.I. Plant polyphenols as dietary antioxidants in human health and disease. Oxid. Med. Cell. Longev. 2009, 2, 270-278. [CrossRef]

4. Cieślik, E.; Grȩda, A.; Adamus, W. Contents of polyphenols in fruit and vegetables. Food Chem. 2006, 94, 135-142. [CrossRef] 
5. Royer, M.; Diouf, P.N.; Stevanovic, T. Polyphenol contents and radical scavenging capacities of red maple (Acer rubrum L.) extracts. Food Chem. Toxicol. 2011, 49, 2180-2188. [CrossRef] [PubMed]

6. Mira, L.; Fernandez, M.T.; Santos, M.; Rocha, R.; Florêncio, M.H.; Jennings, K.R. Interactions of flavonoids with iron and copper ions: A mechanism for their antioxidant activity. Free Radic. Res. 2002, 36, 1199-1208. [CrossRef]

7. Morel, I.; Lescoat, G.; Cillard, P.; Cillard, J. Role of flavonoids and iron chelation in antioxidant action. Methods Enzymol. 1994, 234, 437-443.

8. Ghosh, D.; McGhie, T.K.; Zhang, J.; Adaim, A.; Skinner, M. Effects of anthocyanins and other phenolics of boysenberry and blackcurrant as inhibitors of oxidative stress and damage to cellular DNA in SH-SY5Y and HL-60 cells. J. Sci. Food Agric. 2006, 86, 678-686. [CrossRef]

9. Hirvonen, T.; Virtamo, J.; Korhonen, P.; Albanes, D.; Pietinen, P. Intake of flavonoids, carotenoids, Vitamins $\mathrm{C}$ and $\mathrm{E}$, and risk of stroke in male smokers. Stroke 2000, 31, 2301-2306. [CrossRef]

10. Harborne, J.B.; Williams, C.A. Advances in flavonoid research since 1992. Phytochemistry 2000, 55, 481-504. [CrossRef]

11. Candiracci, M.; Piatti, E.; Dominguez-Barragán, M.; García-Antrás, D.; Morgado, B.; Ruano, D.; Gutiérrez, J.F.; Parrado, J.; Castaño, A. Anti-inflammatory activity of a honey flavonoid extract on lipopolysaccharide-activated N13 microglial cells. J. Agric. Food Chem. 2012, 60, 12304-12311. [CrossRef] [PubMed]

12. Cheng, Y.-C.; Sheen, J.-M.; Hu, W.L.; Hung, Y.-C. Polyphenols and Oxidative Stress in Atherosclerosis-Related Ischemic Heart Disease and Stroke. Oxid. Med. Cell. Longev. 2017. [CrossRef]

13. Ebrahimi, A.; Schluesener, H. Natural polyphenols against neurodegenerative disorders: Potentials and pitfalls. Ageing Res. Rev. 2012, 11, 329-345. [CrossRef] [PubMed]

14. Zhou, Y.; Zheng, J.; Li, Y.; Xu, D.P.; Li, S.; Chen, Y.M.; Li, H. Bin Natural polyphenols for prevention and treatment of cancer. Nutrients 2016, 8. [CrossRef]

15. Blennow, K.; de Leon, M.J.; Zetterberg, H. Alzheimer's disease. Lancet 2006, 368, 387-403. [CrossRef]

16. Karran, E.; Mercken, M.; Strooper, B. De The amyloid cascade hypothesis for Alzheimer's disease: An appraisal for the development of therapeutics. Nat. Rev. Drug Discov. 2011, 10, 698-712. [CrossRef] [PubMed]

17. Serpell, L.C. Alzheimer's amyloid fibrils: Structure and assembly. Biochim. Biophys. Acta Mol. Basis Dis. 2000, 1502, 16-30. [CrossRef]

18. Mattson, M.P. Pathways towards and away from Alzheimer's disease. Nature 2004, 430, 631-639. [CrossRef] [PubMed]

19. Zhao, L.N.; Long, H.; Mu, Y.; Chew, L.Y. The toxicity of amyloid $\beta$ oligomers. Int. J. Mol. Sci. 2012, 13, 7303-7327. [CrossRef]

20. Velander, P.; Wu, L.; Henderson, F.; Zhang, S.; Bevan, D.R.; Xu, B. Natural product-based amyloid inhibitors. Biochem. Pharmacol. 2017, 139, 40-55. [CrossRef]

21. Ono, K.; Yoshiike, Y.; Takashima, A.; Hasegawa, K.; Naiki, H.; Yamada, M. Potent anti-amyloidogenic and fibril-destabilizing effects of polyphenols in vitro: Implications for the prevention and therapeutics of Alzheimer's disease. J. Neurochem. 2003, 87, 172-181. [CrossRef]

22. Korshavn, K.J.; Jang, M.; Kwak, Y.J.; Kochi, A.; Vertuani, S.; Bhunia, A.; Manfredini, S.; Ramamoorthy, A.; Lim, M.H. Reactivity of Metal-Free and Metal-Associated Amyloid- $\beta$ with Glycosylated Polyphenols and Their Esterified Derivatives. Sci. Rep. 2015, 5, 17842. [CrossRef] [PubMed]

23. Ono, K.; Hasegawa, K.; Naiki, H.; Yamada, M. Curcumin Has Potent Anti-Amyloidogenic Effects for Alzheimer's $\beta$-Amyloid Fibrils In Vitro. J. Neurosci. Res. 2004, 75, 742-750. [CrossRef]

24. Ono, K.; Hasegawa, K.; Naiki, H.; Yamada, M. Anti-amyloidogenic activity of tannic acid and its activity to destabilize Alzheimer's $\beta$-amyloid fibrils in vitro. Biochim. Biophys. Acta Mol. Basis Dis. 2004, 1690, $193-202$. [CrossRef] [PubMed]

25. Palhano, F.L.; Lee, J.; Grimster, N.P.; Kelly, J.W. Toward the molecular mechanism(s) by which EGCG treatment remodels mature amyloid fibrils. J. Am. Chem. Soc. 2013, 135, 7503-7510. [CrossRef] [PubMed] 
26. Rivière, C.; Richard, T.; Quentin, L.; Krisa, S.; Mérillon, J.M.; Monti, J.P. Inhibitory activity of stilbenes on Alzheimer's $\beta$-amyloid fibrils in vitro. Bioorganic Med. Chem. 2007, 15, 1160-1167. [CrossRef] [PubMed]

27. Garcia-Alloza, M.; Borrelli, L.A.; Rozkalne, A.; Hyman, B.T.; Bacskai, B.J. Curcumin labels amyloid pathology in vivo, disrupts existing plaques, and partially restores distorted neurites in an Alzheimer mouse model. J. Neurochem. 2007, 102, 1095-1104. [CrossRef] [PubMed]

28. Karuppagounder, S.S.; Pinto, J.T.; Xu, H.; Chen, H.L.; Beal, M.F.; Gibson, G.E. Dietary supplementation with resveratrol reduces plaque pathology in a transgenic model of Alzheimer's disease. Neurochem. Int. 2009, 54, 111-118. [CrossRef] [PubMed]

29. Rezai-Zadeh, K.; Arendash, G.W.; Hou, H.; Fernandez, F.; Jensen, M.; Runfeldt, M.; Shytle, R.D.; Tan, J. Green tea epigallocatechin-3-gallate (EGCG) reduces $\beta$-amyloid mediated cognitive impairment and modulates tau pathology in Alzheimer transgenic mice. Brain Res. 2008, 1214, 177-187. [CrossRef] [PubMed]

30. Xie, L.; Guo, Y.; Cai, B.; Yang, J. Epimerization of epigallocatechin gallate to gallocatechin gallate and its anti-diabetic activity. Med. Chem. Res. 2013, 22, 3372-3378. [CrossRef]

31. Grelle, G.; Otto, A.; Lorenz, M.; Frank, R.F.; Wanker, E.E.; Bieschke, J. Black tea theaflavins inhibit formation of toxic amyloid- $\beta$ and $\alpha$-synuclein fibrils. Biochemistry 2011, 50, 10624-10636. [CrossRef] [PubMed]

32. Vestergaard, M.; Kerman, K. Analytical Tools for Detecting Amyloid Beta Oligomerisation and Assembly. Curr. Pharm. Anal. 2009, 5, 229-245. [CrossRef]

33. Phan, H.T.T.; Shimokawa, N.; Sharma, N.; Takagi, M.; Vestergaard, M.C. Strikingly different effects of cholesterol and 7-ketocholesterol on lipid bilayer-mediated aggregation of amyloid beta (1-42). Biochem. Biophys. Rep. 2018, 14, 98-103. [CrossRef] [PubMed]

34. Vestergaard, M.; Kerman, K.; Tamiya, E. An electrochemical approach for detecting copper-chelating properties of flavonoids using disposable pencil graphite electrodes: Possible implications in copper-mediated illnesses. Anal. Chim. Acta 2005, 538, 273-281. [CrossRef]

35. Vestergaard, M.; Kerman, K.; Saito, M.; Nagatani, N.; Takamura, Y.; Tamiya, E.; Vestergaard, M.; Kerman, K.; Saito, M.; Nagatani, N.; et al. Communication A Rapid Label-Free Electrochemical Detection and Kinetic Study of Alzheimer's Amyloid Beta Aggregation. J. Am. Chem. Soc. 2005, 127, 11892-11893. [CrossRef]

36. Biancalana, M.; Koide, S. Molecular mechanism of Thioflavin-T binding to amyloid fibrils. Biochim. Biophys. Acta Proteins Proteomics 2010, 1804, 1405-1412. [CrossRef]

37. Morita, M.; Hamada, T.; Tendo, Y.; Hata, T.; Vestergaard, M.C.; Takagi, M. Selective localization of Alzheimer's amyloid beta in membrane lateral compartments. Soft Matter 2012, 2012, 2816-2819. [CrossRef]

38. Jiao, Y.; Yang, P. Mechanism of copper(II) inhibiting Alzheimer's amyloid beta-peptide from aggregation: A molecular dynamics investigation. J. Phys. Chem. B 2007, 111, 7646-7655. [CrossRef] [PubMed]

39. Mold, M.; Ouro-Gnao, L.; Wieckowski, B.M.; Exley, C. Copper prevents amyloid- $\beta 1-42$ from forming amyloid fibrils under near-physiological conditions in vitro. Sci. Rep. 2013, 3, 1256. [CrossRef] [PubMed]

40. Ehrnhoefer, D.E.; Bieschke, J.; Boeddrich, A.; Herbst, M.; Masino, L.; Lurz, R.; Engemann, S.; Pastore, A.; Wanker, E.E. EGCG redirects amyloidogenic polypeptides into unstructured, off-pathway oligomers. Nat. Struct. Mol. Biol. 2008, 15, 558-566. [CrossRef]

41. Suprun, E.V.; Khmeleva, S.A.; Radko, S.P.; Kozin, S.A.; Archakov, A.I.; Shumyantseva, V.V. Direct electrochemical oxidation of amyloid- $\beta$ peptides via tyrosine, histidine, and methionine residues. Electrochem. Commun. 2016, 65, 53-56. [CrossRef]

42. Corduneanu, O.; Janeiro, P.; Brett, A.M.O. On the electrochemical oxidation of resveratrol. Electroanalysis 2006, 18, 757-762. [CrossRef]

43. Wu, Y.; Li, W.; Xu, Y.; Jin, E.; Tu, Y. Evaluation of the antioxidant effects of four main theaflavin derivatives through chemiluminescence and DNA damage analyses. J. Zhejiang Univ. Sci. B 2011, 12, 744-751. [CrossRef] [PubMed]

44. Bieschke, J.; Russ, J.; Friedrich, R.P.; Ehrnhoefer, D.E.; Wobst, H.; Neugebauer, K.; Wanker, E.E. EGCG remodels mature $\alpha$-synuclein and amyloid- $\beta$ fibrils and reduces cellular toxicity. Proc. Natl. Acad. Sci. USA 2010, 107, 7710-7715. [CrossRef] [PubMed] 
45. Feng, Y.; Wang, X.P.; Yang, S.G.; Wang, Y.J.; Zhang, X.; Du, X.T.; Sun, X.X.; Zhao, M.; Huang, L.; Liu, R.T. Resveratrol inhibits beta-amyloid oligomeric cytotoxicity but does not prevent oligomer formation. Neurotoxicology 2009, 30, 986-995. [CrossRef]

46. Ladiwala, A.R.A.; Lin, J.C.; Bale, S.S.; Marcelino-Cruz, A.M.; Bhattacharya, M.; Dordick, J.S.; Tessier, P.M. Resveratrol selectively remodels soluble oligomers and fibrils of amyloid $A \beta$ into off-pathway conformers. J. Biol. Chem. 2010, 285, 24228-24237. [CrossRef] [PubMed]

47. Mousseau, N.; Derreumaux, P. Exploring the early steps of amyloid peptide aggregation by computers. Acc. Chem. Res. 2005, 38, 885-891. [CrossRef] [PubMed]

(C) 2019 by the authors. Licensee MDPI, Basel, Switzerland. This article is an open access article distributed under the terms and conditions of the Creative Commons Attribution (CC BY) license (http://creativecommons.org/licenses/by/4.0/). 